\title{
Search for hadronic resonances at CMS
}

\author{
A. Hinzmann* \\ On behalf of the CMS Collaboration \\ CERN, Switzerland \\ E-mail: hinzmannecern.ch
}

Recent results from CMS on searches for new heavy resonances decaying into hadronic final states are summarized. A variety of final states ranging from two jets up to six jets have been analyzed using the data samples of $5 \mathrm{fb}^{-1}$ of pp collisions at $\sqrt{s}=7 \mathrm{TeV}$ and $4 \mathrm{fb}^{-1}$ at $\sqrt{s}=8$ $\mathrm{TeV}$ collected with CMS in 2011 and 2012. In addition to the resonance production of final states with jets from light quarks and gluons, also final states enriched with b-quarks and hadronically decaying $\mathrm{W}$ or $\mathrm{Z}$ bosons are examined using $\mathrm{b}$-tagging and jet substructure techniques.

36th International Conference on High Energy Physics,

July 4-11, 2012

Melbourne, Australia

${ }^{*}$ Speaker. 


\section{Introduction}

CMS [1] has an extensive program of analyses searching for new heavy hadronic resonances [27]. These analyses search for narrow resonances on top of a smoothly falling spectrum of QCD interactions predicted by the Standard Model. Figure 1 summarizes the hadronic final states covered by the analyses presented in this contribution. Both s-channel and pair production of resonances decaying into final states with two to six jets are considered. Additional searches with jets from b-quarks and hadronic $\mathrm{W}$ or $\mathrm{Z}$ boson decays are performed as well. The resonance signal models considered and the exclusion limits set on them are summarized in Table 1. Note that searches with top quark decays and non-resonant signals like contact interactions and black holes are not covered in this contribution and can be found in related contributions to these proceedings.
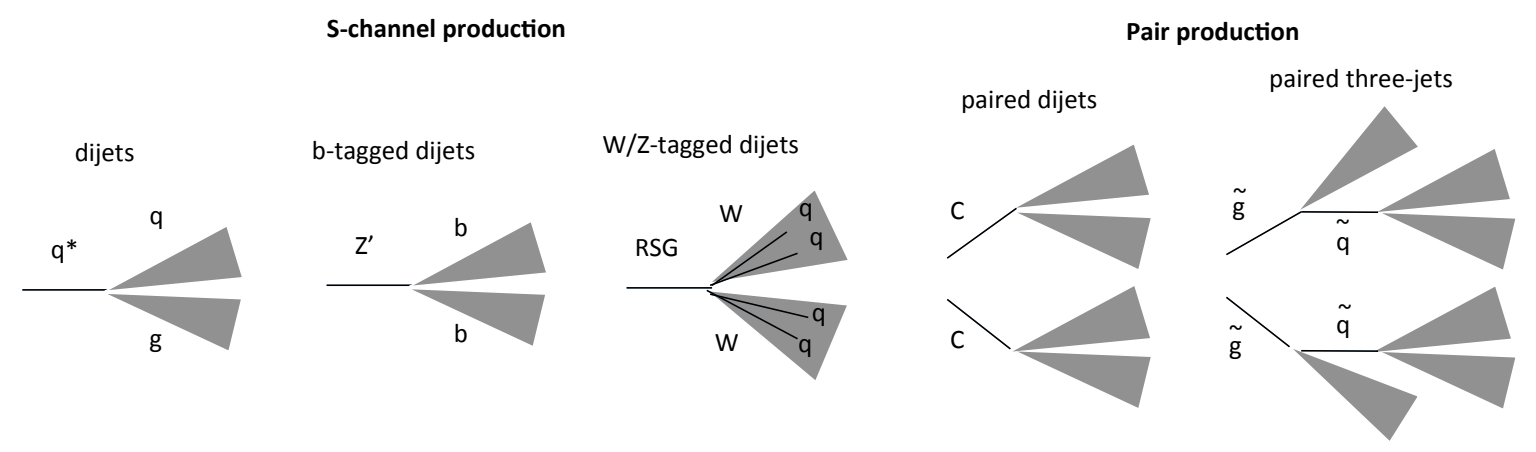

Figure 1: Graphical representation of the final states considered in the analyses discussed in this contribution.

\begin{tabular}{lll}
\hline Model & Final state & Observed exclusion [TeV] \\
\hline String resonances & qg [2,4] & {$[0.6-4.69]$} \\
Excited quarks & qg [2,4],qW,qZ [5] & qq: $[0.6-3.19] \mathrm{qW}:[1.0-2.32] \mathrm{qZ}:[1.0-2.04]$ \\
Scalar diquarks & qq $[2,4]$ & {$[0.6-4.28]$} \\
Axigluons & qq [2,4] & {$[0.6-3.28]$} \\
Color-octet colorons & qq [2,4], qqqq [6] & qq: $[0.6-3.28] \mathrm{qqqq}:[0.32-0.58]$ \\
Color-octet scalar & gg [2,4],bb [3] & qq: $[0.6-2.66] \mathrm{bb}:[1.0-1.08,1.12-1.56]$ \\
W' bosons & qq [2-4],WZ [5] & qq: $[0.6-0.69,0.82-0.96,1.0-1.78,1.97-2.12]$ \\
Z' bosons & qq [2],bb [3] & qq: $[1.0-1.60] \mathrm{bb}:[1.04-1.49]$ \\
RPV SUSY gluinos & qqqqqq [7] & {$[0.28-0.46]$} \\
RS gravitons & qq,gg [2],WW,ZZ [5] & qq+gg: $[1.0-1.36]$ \\
\hline
\end{tabular}

Table 1: Resonance signal models and observed limits on their mass.

\section{Jet reconstruction in CMS}

CMS applies the particle-flow technique to reconstruct jets [1,8]. The particle-flow event reconstruction consists in reconstructing and identifying muons, electrons, photons, charged hadrons 
and neutral hadrons with an optimized combination of all subdetector information. These particles are clustered into jets using the anti- $k_{T}$ algorithm with an $\mathrm{R}$ parameter of 0.5 . The jet energy is calibrated to typically $1-2 \%$ accuracy for jets with $p_{T}>100 \mathrm{GeV}$ with a resolution of $\sim 10 \%$. Energy from additional interactions in the same bunch crossing (pileup) is removed from the jet energies using two techniques. Firstly, charged particles not originating from the primary vertex are excluded from the jet clustering (not used in all presented analyses), and secondly, the jet energy is corrected based on an event energy density and the jet area (used in all presented analyses). The data for the analyses below is taken with triggers requiring multiple jets, typically based on $H_{T}=\sum_{\text {jets }} p_{T}\left(p_{T}>40 \mathrm{GeV}\right)$, and are $>99.9 \%$ efficient above a certain threshold.

\section{Search in dijets}

CMS has performed a search for resonances in dijets using $5 \mathrm{fb}^{-1}$ of data collected at $\sqrt{s}=7$ $\mathrm{TeV}$ [3] and $4 \mathrm{fb}^{-1}$ at $\sqrt{s}=8 \mathrm{TeV}$ [2]. The search is designed in a model independent way and applies to any narrow resonance produced in the s-channel and decaying into a qq, qg or gg final state. The two main kinematic requirements are a dijet invariant mass reconstructed from the two leading jets of larger than $0.89 \mathrm{TeV}$ which is driven by the trigger requirement, and cuts on the jet pseudorapidities $\left|\eta_{1,2}\right|<2.5$ and $\left|\eta_{1}-\eta_{2}\right|<1$.3. The second requirement effectively selects s-channel like scattering while rejecting the QCD background which has an angular distribution similar to t-channel scattering. In order to pick up also the final state radiation of the resonance decays, a wide jet algorithm is applied on the two leading anti- $k_{T}$ jets, merging them with their surrounding jets with $p_{T}>30 \mathrm{GeV}$ and within $\Delta R<1.1$, resulting in a better resonance mass resolution.

In order to investigate also the mass range below $1 \mathrm{TeV}$, a special analysis strategy was developed in 2011 [4]. A lower jet trigger threshold to reach dijet masses down to $0.6 \mathrm{TeV}$ leads to a high rate of events $\left({ }^{\circ H z}\right)$. To get the bandwidth under control, the data format was reduced to jets reconstructed at trigger level with slightly reduced resolution w.r.t. the full reconstruction, however, sufficient for a resonance search. CMS uses this approach for searches in regions of phase space otherwise inaccessible due to trigger constraints.

The background is estimated from a signal+background fit to the smoothly falling QCD spectrum with the function $\frac{\mathrm{d} \sigma}{\mathrm{d} m}=\frac{P_{0}(1-m / \sqrt{s})^{P_{1}}}{(m / \sqrt{s})^{P_{2}+P_{3} \ln (m / \sqrt{s})}}$ and limits are set on the cross section of qq, qg and gg resonances as a function of resonance mass as shown in Figure $2(a, b)$. From these, resonance mass limits on a set of reference models, listed in Table 1, are set.

\section{Search in b-tagged dijets}

By introducing b-tagging into the dijet search [3], the QCD background can be reduced by up to a factor 50 w.r.t. signals with bb final states. A b-tagging algorithm with about $50 \%$ btagging efficiency using variables from reconstructed secondary vertices together with track-based lifetime information is used to identify jets originating from b-quarks. The search is performed simultaneously on dijet mass spectra with exactly 0,1 and $2 \mathrm{~b}$-tags. The cross section limits are presented as a function of the branching ratio of the resonance into qq, gg and bb final states. An example is presented in Figure 2 (c). 


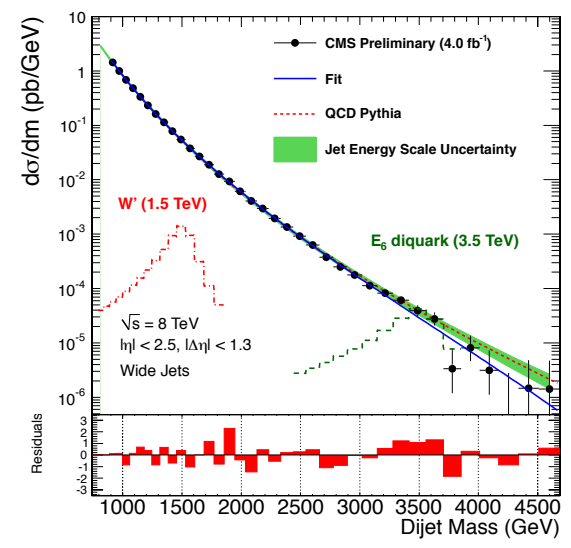

(a)

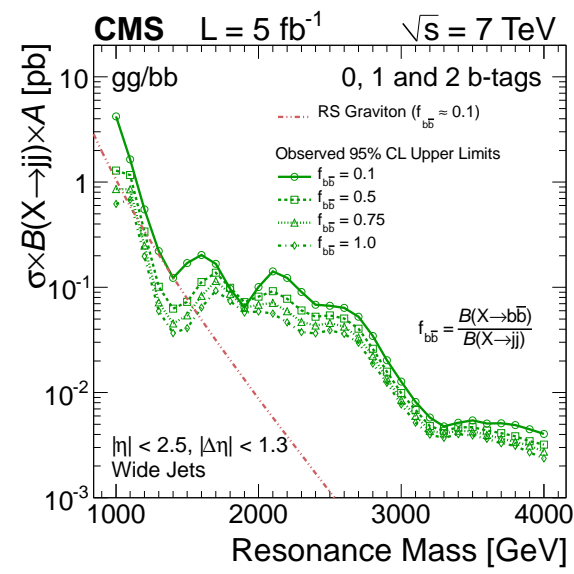

(c)

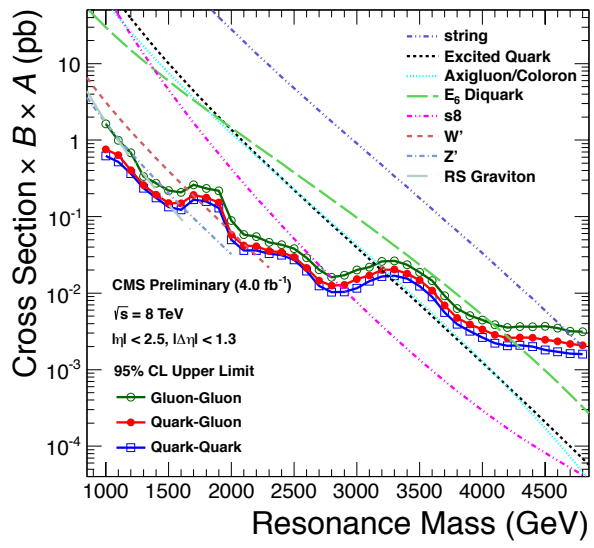

(b)

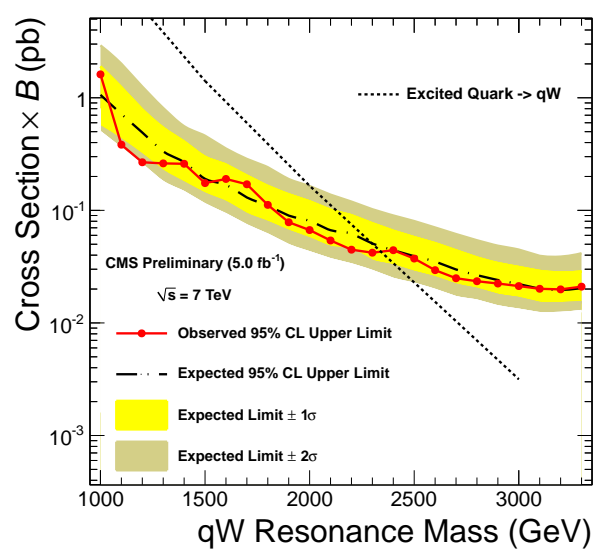

(d)

Figure 2: (a) The dijet mass spectrum in $\sqrt{s}=8 \mathrm{TeV}$ data compared to QCD simulation and a backgroundonly fit. (b) Exclusion limits on qq resonances extracted from (a). (c) Exclusion limits from the b-tagged dijet search as a function of the resonance branching ratio into gg and bb. (d) Exclusion limits from the search in the single W/Z-tagged dijet mass spectrum on $\mathrm{qW}$ resonances.

\section{Search in W/Z-tagged dijets}

The dijet mass spectrum can also be used to search for resonances decaying into boosted hadronic W/Z final states, namely qW, qZ, WW, WZ and ZZ [5]. Above $1 \mathrm{TeV}$ resonance mass, for a high fraction of the produced $\mathrm{W}$ or $\mathrm{Z}$ bosons, the decay products merge into a single jet. Making use of jet substructure techniques, one can tag these boosted hadronic W/Z-bosons. The tagging algorithm is based on the jet mass and the so called mass drop describing the ratio of the jet mass and the mass of the jet split up into two subjets. A pruning algorithm is used to remove soft and large angle particles during the jet clustering which significantly improves the separation power of the jet mass. A working point with a tagging efficiency of $10-30 \%$ is chosen allowing to suppress the QCD background by up to a factor 150 w.r.t. signals with WW final states. Cross section limits are set on qW/qZ resonances in a single-tagged sample and on WW/WZ/ZZ resonances in a double-tagged sample. An example is presented in Figure 2 (d). The search extends the 
corresponding semi-leptonic searches discussed in a related contribution to these proceedings to higher masses.

\section{Search in paired dijets}

Pair production of resonances decaying to dijets is examined in a search in the four jet final state [6]. Three combinations of pairs of dijets are constructed from the four leading jets which are required to have $p_{T}>140 \mathrm{GeV}$ driven by the trigger requirement. For each event, the combination with the smallest $\Delta m / m_{\text {avg }}$ between the two dijets is selected and contributes to the paired dijet average mass distribution shown in Figure 3 (a). To reduce the QCD background, only pairs with $\Delta R_{j j}>0.7, \Delta m / m_{\text {avg }}<0.15$ and $\sum_{i=1}^{2} p_{T}^{i}-m_{\text {avg }}>25 \mathrm{GeV}$ are accepted. The latter cut is applied to remove an infrared singularity in the QCD amplitude. Cross section limits are set on pair produced resonances decaying to two jets and mass limits are set on a benchmark model with pair production of colorons decaying to qq pairs (see Figure 3 (b)).

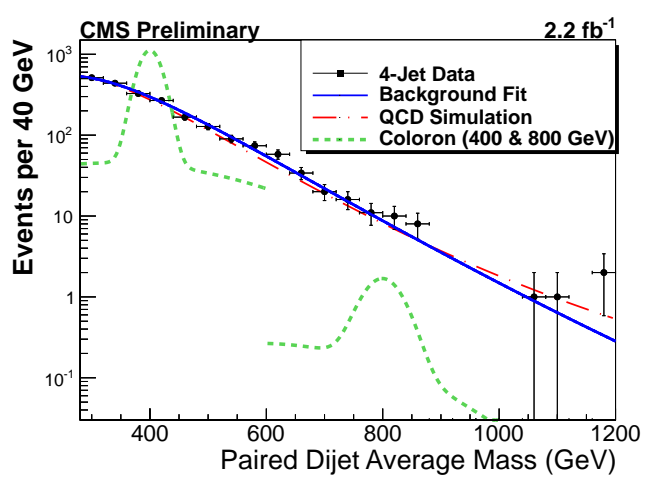

(a)

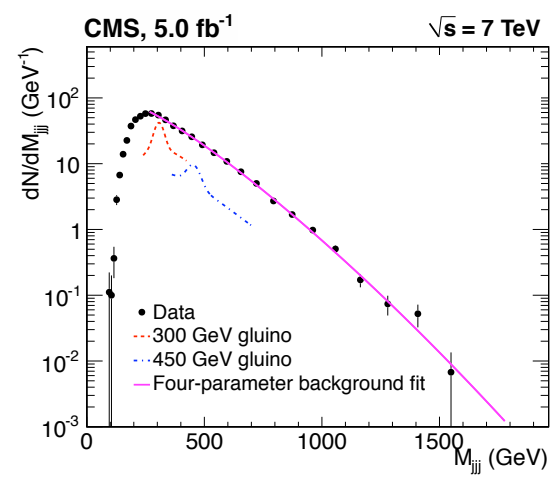

(c)

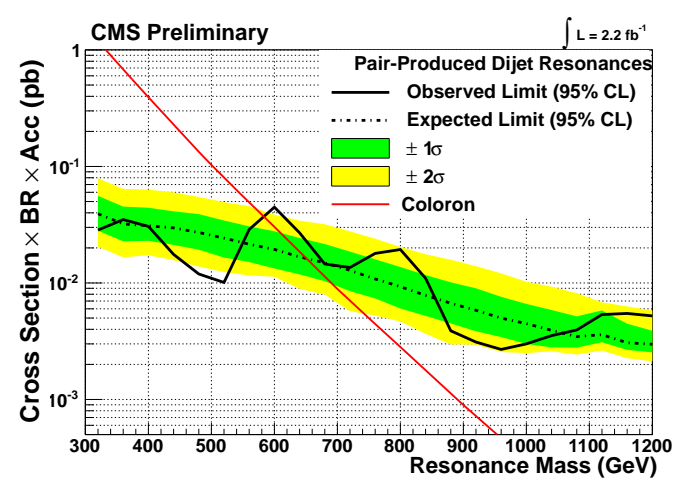

(b)

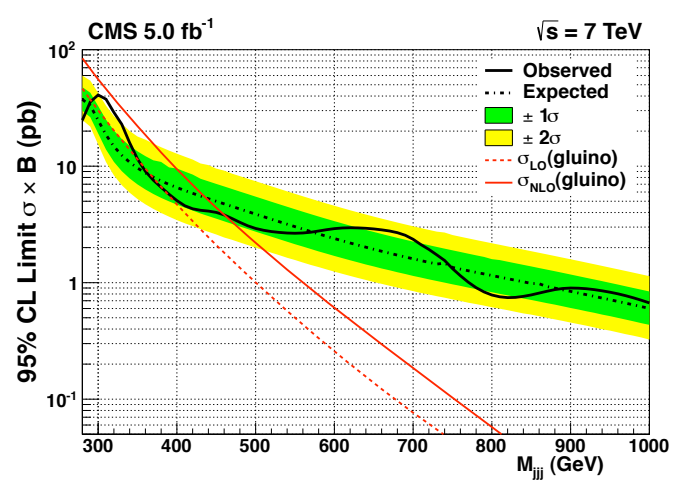

(d)

Figure 3: (a) Paired dijet mass spectrum. (b) Exclusion limits on pair produced resonances decaying to dijets extracted from (a). (c) Three-jet mass spectrum. (d) Exclusion limits on pair produced resonances decaying to three jets extracted from (c).

\section{Search in paired three-jets}

Pair production of resonances decaying to three jets is examined in a search in the six jet 
final state [7]. Twenty combinations of pairs of three-jets are constructed from the six leading jets which are required to have $p_{T}>70 \mathrm{GeV}$ and $H_{T}>900 \mathrm{GeV}$ driven by the trigger requirement. Here the main challenge is to deal with the combinatorial background of three-jet pairs. A jet ensemble technique is used, where all combinations passing certain quality requirements are entries to the three-jet mass distribution shown in Figure 3 (c). Both combinatorial and QCD backgrounds are reduced by a requirement on $\sum_{i=1}^{3} p_{T}^{i}-M_{j j j}>160 \mathrm{GeV}$. Cross section limits are set on pair produced resonances decaying to three jets and mass limits are set on a benchmark model with pair production of RPV SUSY gluinos decaying to qqq triplets (see Figure 3 (d)).

\section{Summary}

A summary of the recent results on hadronic resonance searches in CMS has been given. A large variety of final states is covered, ranging from two to six jet final states. Techniques to reject the QCD and combinatorial backgrounds have been discussed, including b-tagging, W/Z-tagging and kinematic requirements. The presented analyses set limits on a large set of models where the majority are the most stringent to date.

\section{References}

[1] CMS Collaboration, The CMS experiment at the CERN LHC, JINST 0803:S08004, 2008

[2] CMS Collaboration, Search for narrow resonances using the dijet mass spectrum in pp collisions at $\operatorname{sqrt}(s)=8 \mathrm{TeV}$, CMS-PAS-EXO-12-016, 2012, http://cdsweb.cern.ch/record/1462265

[3] CMS Collaboration, Search for narrow resonances and quantum black holes in inclusive and b-tagged dijet mass spectra in pp collisions at sqrt( $(s)=7 \mathrm{TeV}$, Submitted to JHEP, 2012, http://arxiv.org/abs/1210.2387

[4] CMS Collaboration, Search for narrow resonances using the dijet mass spectrum in pp collisions at sqrt(s)=7 TeV, CMS-PAS-EXO-11-094, 2012, http://cdsweb.cern.ch/record/1461223

[5] CMS Collaboration, Search for $q$ W/qZ/WW/WZ/ZZ-resonances in the W/Z-tagged dijet mass spectrum from $7 \mathrm{TeV}$ pp collisions at CMS, CMS-PAS-EXO-11-095, 2012, http://cdsweb.cern.ch/record/1458050

[6] CMS Collaboration, Search for new physics in the paired dijet mass spectrum, CMS-PAS-EXO-11-016, 2012, http://cdsweb.cern.ch/record/1416058

[7] CMS Collaboration, Search for three-jet resonances in pp collisions at sqrt( $(s)=7 \mathrm{TeV}$, Phys. Let. B 10 048, 2012, http://dx.doi.org/10.1016/j.physletb.2012.10.048

[8] CMS Collaboration, Determination of jet energy calibration and transverse momentum resolution in CMS, JINST 6 P11002, 2011, http://dx.doi.org/10.1088/1748-0221/6/11/P11002 